Chronic Obstructive Pulmonary Diseases: Journal of the COPD Foundation

\author{
Original Research
}

\title{
Free Urinary Desmosine and Isodesmosine as COPD Biomarkers: The Relevance of Confounding Factors
}

Sara Ongay, $\mathrm{PhD}^{1}$ Marijke Sikma ${ }^{1,2}$ Peter Horvatovich, $\mathrm{PhD}^{1}$ Jos Hermans ${ }^{1}$ Bruce E. Miller, $\mathrm{MD}, \mathrm{PhD}^{4}$ Nick H.T. ten Hacken, $\mathrm{MD}, \mathrm{PhD}^{3}$ Rainer Bischoff, $\mathrm{PhD}^{1}$

\section{Abstract}

Background: Desmosine (DES) and isodesmosine (IDES) have been widely discussed as potential biomarkers of COPD. However, their clinical utility and validity remains unproven.

Aim: This study aims to progress DES/IDES evaluation as a chronic obstructive pulmonary disease (COPD) biomarker by investigating its urinary excretion in a large sample cohort with respect to a) which factors influence DES/IDES levels in a population of healthy control individuals and COPD individuals; b) whether DES/IDES levels enable the differentiation between COPD individuals and healthy control individuals; c) whether DES/IDES can be used to differentiate between fast and slow decliners in lung function.

Methods: Urinary DES and IDES were quantified in 365 individuals (147 healthy control individuals and 218 COPD individuals) from the Evaluation of COPD Longitudinally to Indentify Predictive Surrogate Endpoints (ECLIPSE) study (NCT00292552) by employing a validated liquid chromatography tandem mass spectrometry (LC-MS/MS) method.

Results: Age, gender, body mass index (BMI) and smoking have a significant $(p<0.05)$ influence on DES/IDES urinary excretion and need to be corrected for when investigating DES/IDES as a disease biomarker. Urinary DES/IDES allowed a statistically relevant differentiation $(p<0.05)$ between stable COPD individuals and healthy control individuals, however, assay sensitivity and specificity were low (62\% and $73 \%$, respectively). Furthermore, urinary DES/IDES does not allow the differentiation of fast and slow decliners in lung function.

Conclusions: The present results suggest that while urinary DES/IDES excretion is related to COPD, it is not a sensitive or specific biomarker for COPD diagnosis or prognosis.

Abbreviations: desmosine, DES; isodesmosine, IDES; chronic obstructive pulmonary disease, COPD; liquid chromatography tandem mass spectrometry, LC-MS/MS; Evaluation of COPD Longitudinally to Identify Predictive Surrogate Endpoints, ECLIPSE; body mass index, BMI; forced expiratory volume in 1 second, FEV $_{\mathbf{1}}$; natural logarithm of desmosine, lnDES; high performance liquid chromatography, HPLC; receiver operator characteristics, ROC

Funding Support: Dutch Technology Foundation (STW) perspectief program P12-04

Date of Acceptance: December 11, 2015

Citation: Ongay S, Sikma M, Horvatovich P, et al. Free urinary desmosine and isodesmosine as COPD biomarkers: The relevance of confounding factors. Chronic Obstr Pulm Dis (Miami). 2016. 3(2):560-569. doi: http://dx.doi.org/10.15326/jcopdf.3.2.2015.0159

\section{This article contains an online supplement.}

1 University of Groningen, Department of Pharmacy, Analytical Biochemistry, Groningen, The Netherlands

2 Van Hall Larenstein Hogeschool, Leeuwarden, Agora, The Netherlands
3 University of Groningen, University Medical Center Groningen, Groningen, The Netherlands

4 GlaxoSmithKline Research and Development, King of Prussia, Pennsylvania 


\section{Address correspondence to:}

Rainer Bischoff, $\mathrm{PhD}$

Phone: +31-50-363-3338 (secr. 3336),

Fax +31-50-363-7582,

Email: r.p.h.bischoff@rug.nl.

\section{Keywords:}

desmosine; isodesmosine; COPD; biomarker; urine; ECLIPSE

\section{Introduction}

Desmosine (DES) and isodesmosine (IDES) are 2 positional isomers that crosslink tropoelastin fibers within mature elastin. ${ }^{1}$ Elastic fibers are present in lung structures including alveoli, alveolar ducts, airways, vasculature and pleura. Thus, when elastin degradation takes place (e.g., as a result of emphysema), DES/IDES (free forms) and DES/IDES-containing peptides (bound forms) are released. ${ }^{2}$ For this reason, the measurement of DES/IDES has been extensively discussed as a potential indicator of elevated lung elastin turnover as well as a potential marker of the effectiveness of agents with the potential to reduce elastic fiber breakdown. ${ }^{3-5}$ This is of particular interest in chronic obstructive pulmonary disease (COPD), a disease characterized by the presence of airflow obstruction mainly caused by chronic bronchitis and/or emphysema. ${ }^{6} \mathrm{Up}$ to now, airflow obstruction reflected by a reduced forced expiratory volume in 1 second $\left(\mathrm{FEV}_{1}\right)$ has been the gold standard marker of COPD progression, response to therapy, and prediction of mortality. ${ }^{7}$ However, it has proven to be a weak indicator of future exacerbations, unable to predict lung function decline as well as ineffective for developing treatment regimes that can significantly reduce mortality rates or alter the disease course. ${ }^{8,9}$ For this reason, there is an increasing need for biomarkers that provide more specific information regarding disease activity and the underlying pathological processes of COPD. ${ }^{10,11}$ In this respect, the development of biomarkers that enable differentiation of individuals with a slow versus fast decline in lung function would be of particular importance for the recognition and treatment of the early stages of the disease. ${ }^{12,13}$ In the last few years, a few studies have been performed in order to investigate the usefulness of urinary DES and IDES in differentiating COPD individuals from healthy control individuals, ${ }^{14-17}$ in differentiating slow from fast decliners in lung function, ${ }^{18,19}$ in predicting COPD exacerbations, ${ }^{20,21}$ as well as in assessing the effect of different treatment regimens on disease progression. ${ }^{22,23}$ However, in spite of the efforts made so far, the clinical utility and validity of urinary DES/ IDES measurements remains unproven and DES/IDES is still far from being considered a reliable biomarker for COPD diagnosis or prognosis. This is due to the lack of standardized methodologies for the quantification of DES and IDES, as well as a lack of consensus on what should be quantified (free or total DES, IDES or DES+IDES) and in which biological fluid (urine, plasma, sputum). In addition, most studies used a low sample size and did not consider possible confounding factors unrelated to COPD. Only the studies of Lindberg et al ${ }^{24}$ and Huang et $\mathrm{al}^{20}$ were performed with more than 50 individuals and Lindberg et al considered confounding factors. There is thus a need for large-scale studies in well characterized samples using fully validated analytical methods in well-matched cohorts to see whether the level of DES/IDES may be used to address relevant questions in COPD. In the present work, we employed a liquid chromatography tandem mass spectrometry (LC-MS/MS) method validated according to the Food and Drug Administration and the European Medicines Agency guidelines for the measurement of free urinary DES/IDES ${ }^{25}$ in 365 individuals from the Evaluation of COPD Longitudinally to Indentify Predictive Surrogate Endpoints (ECLIPSE) cohort (NCT00292552). ${ }^{26}$ The aims of the present work were to investigate whether a) there are confounding factors influencing urinary DES/IDES levels in a population of smoking and nonsmoking individuals with and without COPD; b) urinary DES/IDES levels enable the differentiation between COPD individuals and healthy control individuals; c) urinary DES/IDES levels can be used to differentiate between COPD individuals with a fast or slow decline in lung function.

\section{Materials and Methods}

\section{Study Population}

Urine samples were obtained from 365 individuals at the 1 year study visit from the ECLIPSE (NCT00292552, GlaxoSmithKline Study No. SCO104960) cohort. ${ }^{26}$ (The ECLIPSE study was conducted in accordance with the Declaration of Helsinki and good clinical practice guidelines and was approved by the relevant ethics and review boards at the participating centers.) These individuals comprise 218 individuals with COPD $(17 \%$ with chronic bronchitis, 125 former smokers and 93 current smokers) and 147 control individuals (48 non- 


\section{Table 1. Characteristics of Individuals Included in the Study}

\begin{tabular}{|c|c|c|c|c|c|}
\hline & \multicolumn{2}{|c|}{ COPD Individuals ( $n=218$ ) } & \multicolumn{3}{|c|}{ Healthy Control Individuals $(n=147)$} \\
\hline & $\begin{array}{l}\text { Current Smokers } \\
\qquad(n=93)\end{array}$ & $\begin{array}{l}\text { Former Smokers } \\
\quad(n=125)\end{array}$ & $\begin{array}{l}\text { Current Smokers } \\
\quad(n=50)\end{array}$ & $\begin{array}{l}\text { Former Smokers } \\
(n=49)\end{array}$ & $\begin{array}{l}\text { Non-smokers } \\
\quad(n=48)\end{array}$ \\
\hline Age, years mean (sd) & $62(7)$ & $64(6)$ & $50(6)$ & $57(9)$ & $59(8)$ \\
\hline Gender, M \% & 68 & 62 & 40 & 59 & 40 \\
\hline BMI, kg/m² mean (sd) & $24(5)$ & $26(5)$ & $26(4)$ & $29(5)$ & $28(7)$ \\
\hline Pack years mean (sd) & $48(20)$ & $41(23)$ & $28(14)$ & $27(17)$ & 0 \\
\hline $\mathrm{FEV}_{1}, \mathrm{~L}$ mean (sd) & $1.3(0.4)$ & $1.3(0.5)$ & $3.4(0.7)$ & $3.3(0.7)$ & $3.2(0.8)$ \\
\hline $\begin{array}{l}\text { Change in } \mathrm{FEV}_{1} \text {, } \\
\mathrm{L} / \text { year mean (sd) }\end{array}$ & $-0.19(0.23)$ & $-0.1(0.2)$ & $-0.2(0.2)$ & $-1.7(0.2)$ & $-0.2(0.2)$ \\
\hline $\begin{array}{l}\text { Change in } \mathrm{FEV}_{1} \text {, } \\
\% / \text { year mean (sd) }\end{array}$ & $-14(18)$ & $-8(16)$ & $-6(8)$ & $-5(6)$ & $-5(6)$ \\
\hline $\begin{array}{l}\% \text { predicted } \mathrm{FEV}_{1} \text {, } \\
\% \text { mean }(\mathrm{sd})\end{array}$ & $46(13)$ & $49(15)$ & $108(9)$ & $109(11)$ & $117(13)$ \\
\hline $\begin{array}{l}\text { Change in } \% \text { predicted } \mathrm{FEV}_{1} \text {, } \\
\% / \text { year mean }(\mathrm{sd})\end{array}$ & $-7(8)$ & $-4(8)$ & $-7(8)$ & $-5(7)$ & $-6(7)$ \\
\hline $\mathrm{FEV}_{1} / \mathrm{FVC}, \%$ mean $(\mathrm{sd})$ & $40(9)$ & $43(11)$ & $78(5)$ & $81(6)$ & $80(5)$ \\
\hline
\end{tabular}

sd= standard deviation

smokers, 49 former smokers, 50 current smokers). A detailed group description can be found in Table 1. COPD individuals were on average slightly older than healthy control individuals as well as comprising more males (64\% versus $46 \%$ ). COPD individuals were current and former smokers, while healthy control individuals were divided into non-, former and current smokers. COPD individuals who reported an exacerbation during the 4 weeks preceding enrollment were excluded. Lung function decline was defined as the change in $\mathrm{FEV}_{1}$ over time expressed as \%/year (change in $\mathrm{FEV}_{1}[\mathrm{~L} /$ year $\left.] / \mathrm{FEV}_{1}[\mathrm{~L}]^{*} 100\right)$.

\section{Determination of Urinary DES and IDES}

Free urinary DES and IDES were determined as described before. ${ }^{25}$ Briefly, DES/IDES was extracted from $0.5 \mathrm{~mL}$ urine using an Oasis HLB solid phase extraction cartridge (Waters, Manchester, UK) and heptafluorobutyric acid as an ion-pairing reagent. Purified DES and IDES were separated by high performance liquid chromatography (HPLC) (Agilent, 1200 series) on a reversed phase column (Atlantis ${ }^{\circledR}$ $\mathrm{dC} 18$, Waters). Both isomers were quantified by selected reaction monitoring on a triple quadrupole mass spectrometer (Agilent, G6410B) in the positive ion mode. $\mathrm{D}_{4}$-DES and $\mathrm{D}_{4}$-IDES were used as internal standards. Urine creatinine was determined with the ADVIA ${ }^{\circledR}$ Chemistry system and the Creatinine_2 test (Siemens, Munich, Germany).

\section{Statistical Methods}

Linear regression was used to investigate the relationship between the natural logarithm of desmosine (lnDES, DES expressed in ng DES/mg creatinine) as dependent variable and the membership of the different individuals to the groups under investigation (current smokers, non-smokers, former smokers, COPD individuals, healthy control individuals, slow decliners, fast decliners; see Table 2 for details) as independent variables. The relationship between the dependent variable and the independent variables was investigated with 2 models. In Model 1, the independent variable (smoking habits, disease status, lung function decline) had 2 categories based on whether it belonged to the group under investigation or not (values of 1 and 0 , respectively). In Model 2, gender, age, body mass index (BMI) and smoking habits (when applicable) were corrected for to account for possible confounding factors. The standardized coefficient $(\beta)$ is indicative of the difference between the mean $\operatorname{lnDES}$ values in the 2 categories of an independent variable. The resulting $p$-values were employed to investigate the 


\section{Table 2. Linear Regression Analysis With InDES Levels (Ng/Mg Creatinine) as Dependent Variable and Different Clinical Parameters as Independent Variables}

\begin{tabular}{|c|c|c|c|}
\hline $\begin{array}{l}\text { Independent } \\
\text { Variable }\end{array}$ & $\begin{array}{l}\text { Dependent Variable: InDES } \\
\text { (mean, standard deviation, number of individuals) }\end{array}$ & $\begin{array}{l}\text { Model } 1 \\
\beta \text { (p-value) }\end{array}$ & $\begin{array}{l}\text { Model } 2 \\
\beta \text { (p-value) }\end{array}$ \\
\hline \multirow{6}{*}{$\begin{array}{l}\text { Smoking } \\
\text { Habits }\end{array}$} & \multicolumn{3}{|l|}{ Controls } \\
\hline & Non- $(1.61,0.38,48)$ vs. former $(1.69,0.37,49)$ smokers & $0.103(0.314)$ & $0.173(0.058)$ \\
\hline & Non- $(1.61,0.38,48)$ vs. current $(1.76,0.29,50)$ smokers & $0.214(0.035)$ & $0.353(0.001)$ \\
\hline & Former $(1.69,0.37,49)$ vs. current $(1.76,0.29,50)$ smokers & $0.103(0.309)$ & $0.149(0.130)$ \\
\hline & \multicolumn{3}{|l|}{ COPD } \\
\hline & Former $(1.88,0.37,125)$ vs. current $(1.94,0.38,93)$ smokers & $0.069(0.308)$ & $0.159(0.017)$ \\
\hline \multirow{4}{*}{$\begin{array}{l}\text { Disease } \\
\text { Status }\end{array}$} & Controls $(1.69,0.35,147)$ vs. COPD $(1.91,0.38,218)^{*}$ & $0.281(4.8 \mathrm{E}-8)$ & $0.165(0.007)$ \\
\hline & $\begin{array}{l}\text { Controls (former + current smokers) }(1.73,0.33,99) \text { vs. COPD } \\
(1.91,0.38,218)\end{array}$ & $0.226(5.1 \mathrm{E}-5)$ & $0.131(0.032)$ \\
\hline & $\begin{array}{l}\text { Controls (current smokers) }(1.76,0.29,50) \text { vs. COPD (current smokers) } \\
(1.94,0.38,93)\end{array}$ & $0.237(0.004)$ & $0.049(0.621)$ \\
\hline & $\begin{array}{l}\text { Controls (former smokers) }(1.69,0.37,49) \text { vs. COPD (former smokers) } \\
(1.88,0.37,125)\end{array}$ & $0.228(0.002)$ & $0.166(0.027)$ \\
\hline \multirow{8}{*}{$\begin{array}{l}\text { Lung } \\
\text { Function } \\
\text { Decline }\end{array}$} & \multicolumn{3}{|l|}{ Controls } \\
\hline & Slow $(-4.1,5.8,128)$ vs. fast decliners $(-15.9,4.6,18)^{*}$ & $-0.018(0.826)$ & $-0.008(0.915)$ \\
\hline & \multicolumn{3}{|l|}{ COPD } \\
\hline & Slow $(3.0,11.5,110)$ vs. fast decliners $(-24.4,8.9,109)^{*}$ & $0.066(0.329)$ & $0.095(0.138)$ \\
\hline & \multicolumn{3}{|l|}{ COPD current smokers } \\
\hline & Slow $(1.9,11.7,39)$ vs. fast decliners $(-26.6,9.5,53)$ & $0.129(0.217)$ & $0.162(0.106)$ \\
\hline & \multicolumn{3}{|l|}{ COPD former smokers } \\
\hline & Slow $(3.6,11.4,69)$ vs. fast decliners $(-21.6,7.9,55)$ & $0.005(0.957)$ & $0.044(0.601)$ \\
\hline
\end{tabular}

\footnotetext{
* In these cases smoking habits are included as correction factors
}

Model 1 , independent variable $=$ individual membership to group under investigation .

Model 2, independent variable $=$ individual membership to group under investigation + gender, age and BMI as correction factors.

Standardized coefficient $(\beta)$ and corresponding regression significance ( $p$-value) are shown.

significance in differentiating between the 2 categories of the independent variable. The influence of possible confounding factors (gender, age, BMI and smoking habits) on lnDES urinary excretion was investigated by linear regression using $\operatorname{lnDES}$ as dependent variable and the confounding factors as independent variables. All statistical analyses, box plots and ROC curves were performed with SPSS (IBM, Statistics 22).

\section{Results}

Urinary DES and IDES levels were measured in 365 individuals including COPD patients (current and former smokers), and healthy control individuals (current, former and non-smokers) with slow or fast decline in lung function. While DES and IDES were quantified separately, we observed a constant DES to IDES ratio of $1.5(n=365, R S D=10 \%)$ for the investigated individuals independent of their health status and decline in lung function in agreement with other studies. ${ }^{19,27}$ We will therefore only refer to the DES data for simplicity reasons. The mean levels of urinary DES in the different groups are given in Table 2.

Based on smoking habits, no statistically relevant differences in lnDES ( $p=0.308$, Table 2 , Model 1) were observed between former and current smokers among the COPD individuals. Statistically relevant differences were observed between non- and current smokers among the healthy control individuals, although significance was low ( $p=0.035$, Table 2, Model 1$)$. DES urinary excretion allowed a statistically significant 
differentiation between COPD individuals and healthy control individuals independent of the smoking habits ( $p=4.8 \times 10^{-8}$, "disease status" in Table 2, Model 1). However, DES urinary levels could not be used to differentiate slow and fast decliners in lung function independently of their health status or smoking habits ( $p>0.05$, "lung function decline" in Table 2, Model 1). Similar results were obtained for different definitions of lung function decline (change in $\mathrm{FEV}_{1}$ [L/year] or change in \% predicted $\mathrm{FEV}_{1}$ [\%/year]).

A previous study showed that age, gender and BMI may have an influence on urinary excretion of DES and illustrated the importance of adequately correcting for these factors (especially age). ${ }^{24}$ Thus, we investigated whether factors such as age, gender, BMI or smoking habits may influence urinary DES excretion and thus need to be corrected for. The influence of age, gender, BMI and smoking habits on urinary DES excretion in individuals with and without COPD was evaluated by multiple linear regression analysis (Table 3). Results show that all of these factors have an influence on urinary DES levels $(p<0.05)$ with a major contribution of age, gender and smoking habits (current versus former and never-smoker).

\section{Table 3. Multiple Linear Regression Analysis of Parameters Potentially Influencing Urinary DES Levels in the Population Under Study}

\begin{tabular}{l|c|c}
$\begin{array}{l}\text { Independent } \\
\text { Variables }\end{array}$ & $\begin{array}{c}\text { Dependent Variable: } \\
\text { InDES }\end{array}$ \\
\hline $\begin{array}{l}\text { Age (year) } \\
\text { Gender } \\
\text { (male } / \text { female) }\end{array}$ & 0.350 & $2.75 \mathrm{E}-12$ \\
\hline BMI (kg/m ) & 0.301 & $4.64 \mathrm{E}-10$ \\
\hline $\begin{array}{l}\text { Current Smoking } \\
\text { (vs. Never Smokers) }\end{array}$ & 0.121 & 0.012 \\
\hline $\begin{array}{l}\text { Former Smoking } \\
\text { (vs. Never Smokers) }\end{array}$ & 0.280 & $7.97 \mathrm{E}-9$ \\
\hline
\end{tabular}

The standardized coefficient $(\beta)$ and corresponding regression significance ( $p$-value) are shown.

$\mathrm{BMI}=$ body mass index
After correcting for confounding factors (gender, age, BMI and smoking habits, Model 2), a statistically relevant difference was observed between COPD current and former smokers ( $p=0.017$, Table 2, Model 2). In healthy control individuals the only statistically relevant difference was observed between non- and current smokers ( $p=0.001$, Table 2, Model 2) as already observed before correcting for gender, age and BMI. After correction for gender, age, BMI and smoking habits, statistically relevant differences were also observed between COPD individuals and healthy control individuals ( $p=0.007$, Table 2, Model 2) although significance was lowered due to the confounding effect of age, gender and BMI on urinary DES excretion. If individuals are grouped based on smoking habits, statistically relevant differences were still observed between COPD individuals and healthy control individuals with the exception of current smokers ( $p=0.621$, Table 2, Model 2 ) due to the general influence of current smoking on DES excretion (Table 3) especially in healthy control individuals (Table 2, compare Models 1 and 2). In spite of the correction for confounding factors, no statistically relevant differences were observed between slow and fast decliners in lung function (lung function decline, Table 2, Model 2). Similar results were obtained for different definitions of lung function decline (change in $\mathrm{FEV}_{1}$ [L/year] or change in \% predicted $\mathrm{FEV}_{1}$ [\%/year]) (Table $1 \mathrm{SI}$ and Figure 1SI in the online supplementary data).

\section{Discussion}

The present study, comprising a large number of COPD individuals and control individuals, shows that a) gender, age, BMI and smoking habits have a strong influence on DES urinary excretion and need to be considered as confounding factors when investigating urinary DES as a disease biomarker; b) urinary DES excretion is related to COPD enabling the differentiation of COPD individuals and healthy control individuals, although assay sensitivity and specificity are low (62\% and $73 \%$, respectively); c) urinary DES excretion cannot be used as a prognostic biomarker to predict the decline of $\mathrm{FEV}_{1}$ in COPD since it does not allow the differentiation between COPD individuals with fast and slow decline in lung function.

In COPD, lung elastin degradation can take place as a consequence of exposure to cigarette smoke, among other factors. $^{28}$ For this reason, it is important to 
investigate whether smoking influences DES urinary excretion and whether smoking habits need to be taken into account when using DES as a COPD biomarker. Our results show a trend towards higher DES values in both control individuals and COPD individuals when comparing non-, former and current smokers (Table 2, Figure 1). However, differences among the groups were only statistically significant in healthy control individuals when comparing current and non-smokers $(p=0.035$, Table 2, Model 1$)$. These results are in line with previous publications reporting a trend towards increased urinary DES/IDES excretion in relation to smoking without reaching statistically significant values. ${ }^{15,20,29}$ Lindberg and co-workers ${ }^{24}$ observed that urinary DES/IDES was significantly associated with smoking habits after correction for age, gender and BMI in 349 individuals. We obtained similar results with a statistically significant difference between former and current smokers among COPD individuals after correction for gender, age and BMI $(p=0.017$, Table
2, Model 2). In the case of healthy control individuals, correction for these factors led to a more significant differentiation between non-smokers and current smokers $(p=0.001)$, between non-smokers and former smokers $(p=0.058)$, and between former and current smokers $(p=0.130)$ albeit that statistical significance $(p<0.05)$ was not reached in the 2 latter cases (Table 2, Model 2). Thus, it can be concluded that current smoking has a considerable effect on urinary DES/IDES excretion (Tables 1 and 2) in COPD individuals and even more so in healthy control individuals. Smoking and COPD are both contributing to elastin degradation, thus, if DES values are not corrected for smoking habits misleading results may be obtained for urinary DES excretion in relation to COPD.

Gender, age, and BMI are further confounding factors influencing urinary DES excretion (Table 3) and need to be taken into account when investigating the utility of urinary DES as a COPD biomarker. Thus, differences between COPD individuals and healthy control
individuals (Figure $2 \mathrm{~A}$ ) were evaluated before

\section{Figure 1. Box Plot Representation of InDES Levels: Based on Smoking Habits}

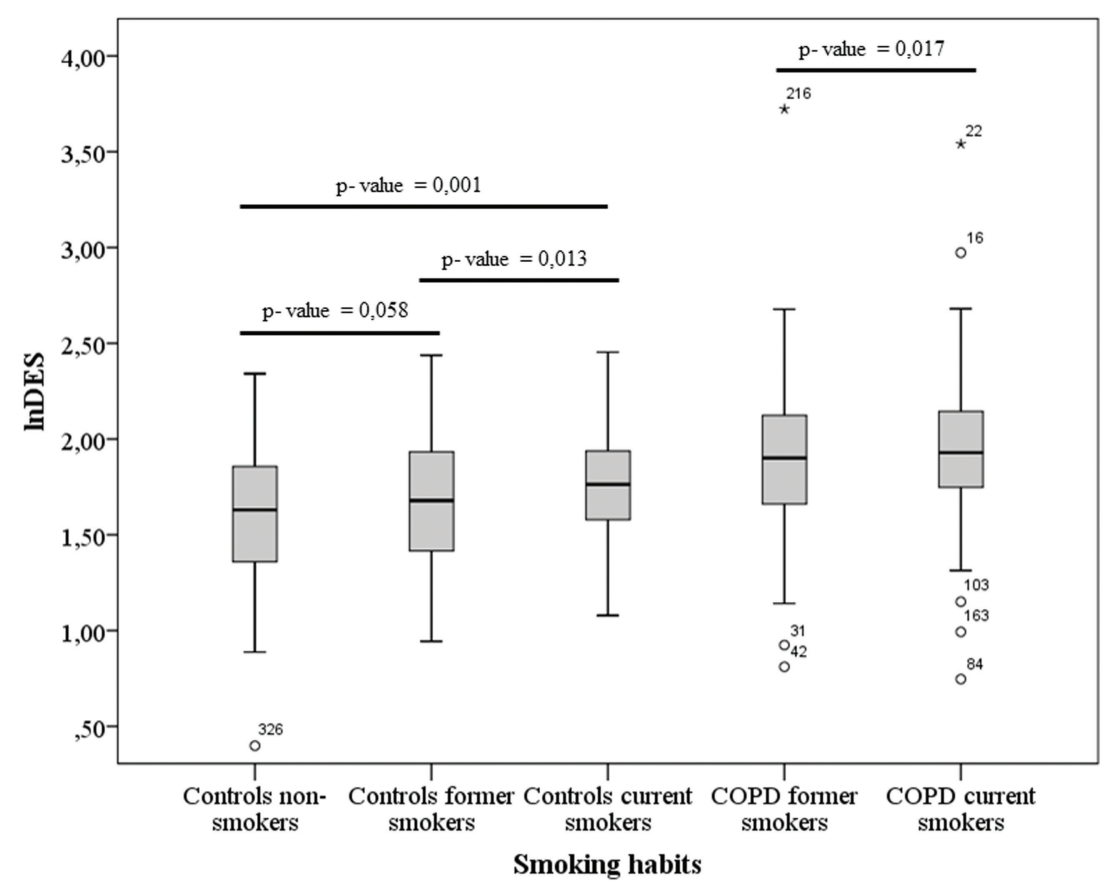

Box plot representation of 1 DDES levels (ng DES/mg creatinine) for healthy control individuals and COPD individuals grouped on the basis of smoking habits (model 2 -based $p$-values are displayed). and after correction for these factors (Table 2, Models 1 and 2, respectively). Our results show statistically significant differences between COPD individuals and healthy control individuals (Table 2). However, it has to be pointed out that the difference between healthy control individuals and COPD individuals is lower after correction for gender, age, BMI and smoking habits due to the additional effect of current smoking on urinary DES excretion. Furthermore, in spite of the statistically relevant difference observed between COPD individuals and healthy control individuals ( $p$-value $=0.007$, Table 2, Model 2), there is considerable overlap between groups (Figure 2A) resulting in an assay with rather low sensitivity and specificity ( $62 \%$ and $73 \%$, respectively, Figure 2B). Previous studies produced contradictory results in this respect. While a few studies observed statistically relevant differences between COPD individuals and healthy control individuals, ${ }^{15,17,27,29}$ Huang and coworkers $^{20}$ reported no elevation of urinary DES in stable COPD (only in COPD during exacerbations). These discrepancies may be due to the high inter-individual variability of urinary DES excretion (Figure 2), the 


\section{Figure 2. Urinary DES Excretion in COPD Individuals and Healthy Control Individuals}

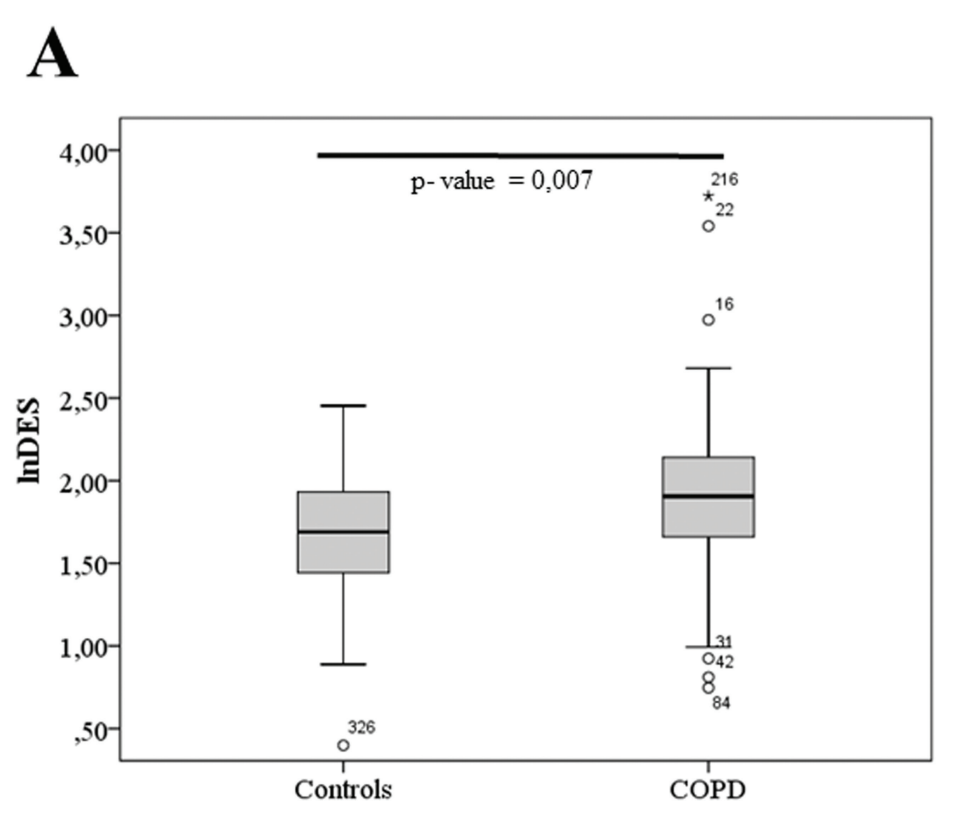

Health status
B

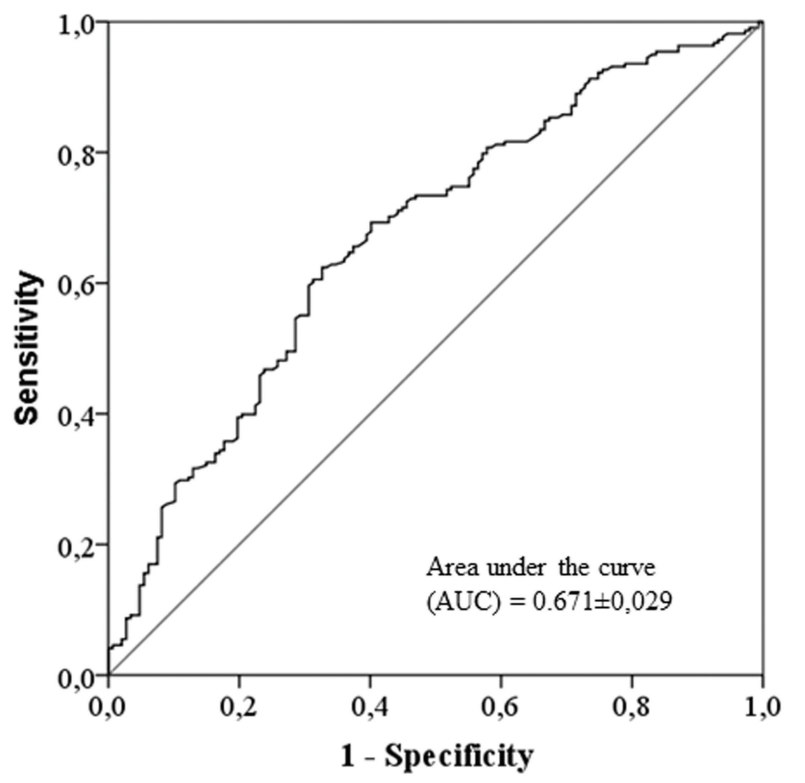

(A) Box plot representation of lnDES levels (ng DES/mg creatinine) in healthy control individuals and COPD individuals (the Model 2-based $p$-value is displayed);

(B) Receiver operator characteristics (ROC) curve for differentiating individuals with COPD from healthy control individuals.

influence of confounding factors, the highly variable excretion of urinary DES over time (especially for COPD individuals), ${ }^{30}$ as well as differences in sample size between studies.

Finally, we investigated the capability of urinary DES to reflect lung function decline by comparing individuals with fast lung function decline (fast decliners, with a decline in $\mathrm{FEV}_{1}$ [\%/year] of more than $12 \%$ ) with those having slow lung function decline (slow decliners, with a decline in $\mathrm{FEV}_{1}$ [\%/year] of less than $12 \%$ ). We observed a trend towards higher DES urinary excretion in COPD fast decliners when compared to COPD slow decliners (Table 2, Figure 3). However, differences were not statistically significant ( $p>0.05$, Table 2$)$, independent of smoking habits. Control individuals even showed a trend towards increasing DES urinary excretion in slow decliners when compared to fast decliners (Table 2, Figure 3), but this effect was not statistically significant ( $p>0.05$, Table 2$)$. In this respect, previous studies showed contradictory results. According to Gottlieb and co-workers, ${ }^{19}$ the mean urinary excretion of DES was 36\% higher (30\% after adjustment for age and lean body mass) in individuals with fast lung function decline $(n=10)$ than in individuals with slow lung function decline $(n=8)$. However, in spite of the observed differences, a large variability of DES excretion in the group of fast decliners resulted in a considerable overlap with the group of slow decliners. On the other hand, Boutin and co-workers ${ }^{18}$ observed a statistically lower excretion of urinary DES in fast decliners $(n=9)$ when compared to slow decliners $(n=11)$. Considering the rather small number of individuals and the large inter-individual variability, it is hard to draw definitive conclusions from these studies.

A potential limitation of our study is the use of singlepoint urine samples, which are less representative than urine collected over 24 hours. In addition, as urinary DES excretion in COPD individuals can vary over time, ${ }^{30}$ assessing DES excretion over the complete 3-year period of the ECLIPSE study might lead to better correlations with lung function decline. However, it was our aim to see whether fast and slow decliners could be discriminated from each other using a single urinary DES measurement, as this is much easier to implement in clinical practice. Another limitation might be not taking into account the effect decreased renal function 


\section{Figure 3. Box Plot Representation of $1 \mathrm{nDES}$ Levels: Based on Lung Function Decline}

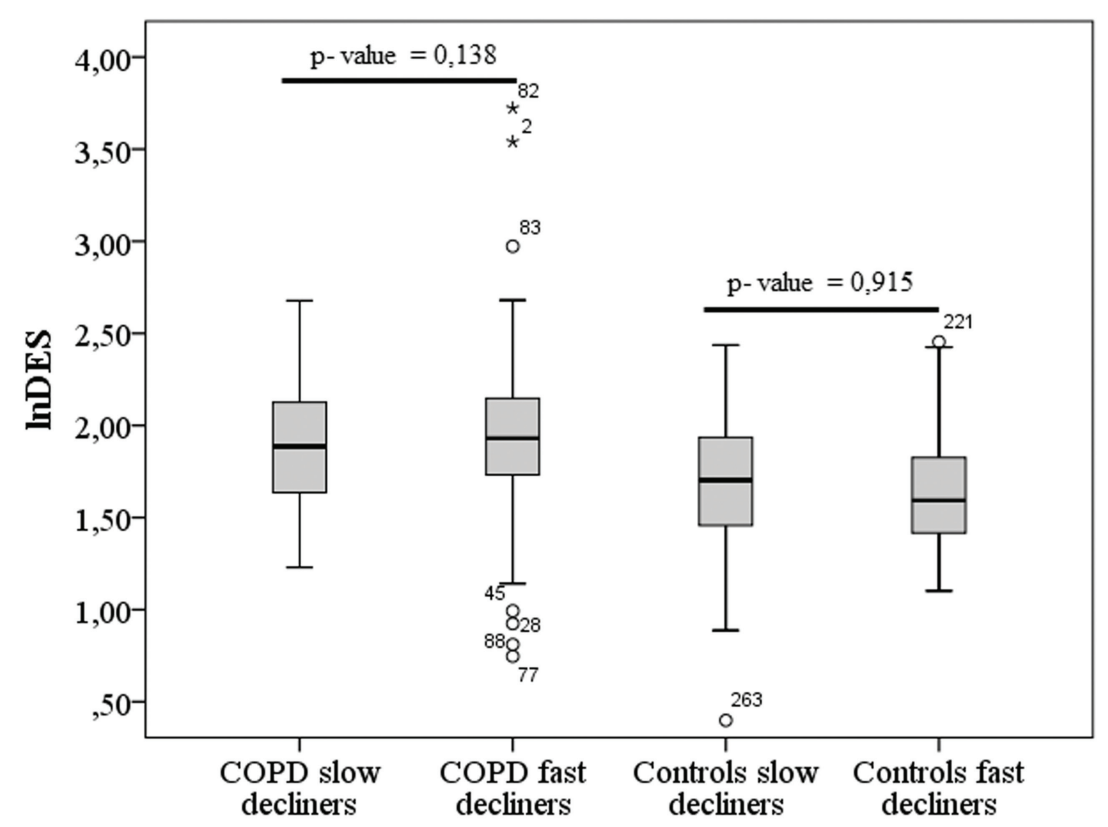

Lung function decline

Box plot representation of 1 DDES levels (ng DES/mg creatinine) for control individuals and COPD individuals grouped on the basis of lung function decline (Model 2-based $p$-values are displayed).

too many COPD patients with rather mild emphysema. The selection of emphysema in our study was based on the ECLIPSE definition, including individuals with more than $10 \%$ of lung volume with a density of -950 Hounsfield units or less during a maximal inspiratory breath hold. ${ }^{32}$ This has led to the rather high $83 \%$ prevalence of emphysema in our COPD group. We further did not observe any association between DES excretion and loss in lung density.

In summary, our study shows that there is no need to quantify DES and IDES separately, as there is a constant ratio between both isomers independent of health status or decline in lung function. Our results corroborate the findings of Lindberg et $\mathrm{al}^{24}$ regarding the effect of gender, age, BMI or smoking habits on urinary DES excretion. After correction for these confounding factors, a statistically significant difference between COPD individuals and healthy control individuals was observed $(p=0.007)$, although current smoking seems to be the main factor affecting DES excretion, as there is no statistically significant difference between current smokers whether they have COPD or not. These results emphasize the need to correct for confounding factors (especially current

has on desmosine excretion into urine. However, close relationships between desmosine concentrations in urine and plasma were reported by Lindberg et al in another comparable COPD cohort. ${ }^{24}$ Furthermore, we expressed desmosine in ng DES / mg creatinine. Finally, within the group of COPD individuals, $83 \%$ were diagnosed as having emphysema, while the rest are individuals in which other pathological processes may lead to a different urinary DES excretion pattern. However, considering only the emphysema group among the COPD individuals did not lead to a statistically significant relation between urinary DES levels and lung function decline. One possible explanation could be that other elastin-containing tissues contribute to desmosine excreted in urine, as only $19 \%$ has been estimated to result from elastin turnover in the lung. ${ }^{31}$ Another possible explanation could be that we did select smoking) when investigating urinary DES excretion as a COPD biomarker. Our data show further that a single measurement of urinary DES excretion cannot predict lung function decline in stable COPD individuals.

\section{Declaration of Interest}

Sara Ongay, Marijke Sikma, Peter Horvatovich, Jos Hermans, Nick H.T. ten Hacken and Rainer Bischoff have no conflicts of interest to declare.

Bruce E. Miller is a current employee and shareholder of GlaxoSmithKline. Dr. Miller was a member of the ECLIPSE Scientific Committee and contributed to the design and interpretation of the ECLIPSE study and the work reported here. 


\section{References}

1. Foster JA, Curtiss SW. The regulation of lung elastin synthesis. Am J Physiol. 1990;259(2):L13-23.

2. Chrzanowski P, Keller S, Cerreta J, Mandl I, Turino GM. Elastin content of normal and emphysematous lung parenchyma. Am J Med. 1980;69(3):351-359. doi: http://dx.doi.org/10.1016/0002-9343(80)90004-2

3. Turino GM, Lin YY, He J, Cantor JO, Ma S. Elastin degradation: An effective biomarker in COPD. COPD. 2012;9(4):435-438. doi: http://dx.doi.org/10.3109/15412555.2012.697753

4. Casaburi R, Celli B, Crapo J, et al. The COPD biomarker qualification consortium (CBQC). COPD. 2013;10(3):367-377. doi: http://dx.doi.org/10.3109/15412555.2012.752807

5. Luisetti M, Stolk J, Iadarola P. Desmosine, a biomarker for COPD: Old and in the way. Eur Respir J. 2012;39(4):797-798. doi: http://dx.doi.org/10.1183/09031936.00172911

6. Viegi G, Pistelli F, Sherrill DL, Maio S, Baldacci S, Carrozzi L. Definition, epidemiology and natural history of COPD. Eur Respir J. 2007;30(5):993-1013. doi: http://dx.doi.org/10.1183/09031936.00082507

7. Kristan SS. Blood specimen biomarkers of inflammation, matrix degradation, angiogenesis, and cardiac involvement: A future useful tool in assessing clinical outcomes of COPD patients in clinical practice? Arch Immunol Ther Exp (Warsz). 2013;61(6):469-481.

doi: http://dx.doi.org/10.1007/s00005-013-0237-y

8. Sin DD, Vestbo J. Biomarkers in chronic obstructive pulmonary disease. Proc Am Thorac Soc. 2009;6(6):543-545. doi: http://dx.doi.org/10.1513/pats.200904-019DS

9. Russell RE. Chronic obstructive pulmonary disease: Getting it right. Does optimal management of chronic obstructive pulmonary disease alter disease progression and improve survival? Curr Opin Pulm Med. 2014;20(2):127-131. doi: http://dx.doi.org/10.1097/mcp.0000000000000030

10. Faner R, Tal-Singer R, Riley JH, et al. Lessons from ECLIPSE: A review of COPD biomarkers. Thorax. 2014;69(7):666-672. doi: http://dx.doi.org/10.1136/thoraxjnl-2013-204778

11. Pelaia G, Busceti MT, Maselli R, et al. Application of proteomics and peptidomics to COPD. Biomed Res Int. 2014; doi: http://dx.doi.org/10.1155/2014/764581

12. Tashkin DP. Variations in $\mathrm{FEV}_{1}$ decline over time in chronic obstructive pulmonary disease and its implications. Curr Opin Pulm Med. 2013;19(2):116-124.

doi: http://dx.doi.org/10.1097/MCP.0b013e32835d8ea4

13. Higashimoto Y, Iwata T, Okada M, Hiroaki S, Fukuda K, Tohda Y. Serum biomarkers as predictors of lung function decline in chronic obstructive pulmonary disease. Respir Med. 2009;103(8):1231-1238.

doi: http://dx.doi.org/10.1016/j.rmed.2009.01.021

14. Albarbarawi O, Barton A, Lin ZS, et al. Measurement of urinary total desmosine and isodesmosine using isotope-dilution liquid chromatography-tandem mass spectrometry. Anal Chem. 2010;82(9):3745-3750. doi: http://dx.doi.org/10.1021/ac100152f
15. Cocci F, Miniati M, Monti S, et al. Urinary desmosine excretion is inversely correlated with the extent of emphysema in patients with chronic obstructive pulmonary disease. Int J Biochem Cell Biol. 2002;34(6):594-604. doi: http://dx.doi.org/10.1016/S1357-2725(02)00015-8

16. Devenport NA, Reynolds JC, Parkash V, Cook J, Weston DJ, Creaser CS. Determination of free desmosine and isodesmosine as urinary biomarkers of lung disorder using ultra performance liquid chromatography-ion mobility-mass spectrometry. $J$ Chromatogr B-Analyt Technol Biomed Life Sci. 2011; 879(32):3797-3801. doi: http://dx.doi.org/10.1016/j.jchromb.2011.10.016

17. Ma S, Turino GM, Lin YY. Quantitation of desmosine and isodesmosine in urine, plasma, and sputum by LC-MS/MS as biomarkers for elastin degradation. J Chromatogr B-Analyt Technol Biomed Life Sci. 2011;879(21):1893-1898. doi: http://dx.doi.org/10.1016/j.jchromb.2011.05.011

18. Boutin M, Berthelette C, Gervais FG, et al. High-sensitivity NanoLC-MS/MS analysis of urinary desmosine and isodesmosine. Anal Chem. 2009;81(5):1881-1887. doi: http://dx.doi.org/10.1021/ac801745d

19. Gottlieb DJ, Stone PJ, Sparrow D, et al. Urinary desmosine excretion in smokers with and without rapid decline of lung function - the normative aging study. Am J Respir Crit Care Med. 1996;154(5):1290-1295.

doi: http://dx.doi.org/10.1164/ajrccm.154.5.8912738

20. Huang JTJ, Chaudhuri R, Albarbarawi O, Barton A, Grierson $C$, Rauchhaus $P$, et al. Clinical validity of plasma and urinary desmosine as biomarkers for chronic obstructive pulmonary disease. Thorax. 2012;67(6):502-8. doi: http://dx.doi.org/10.1136/thoraxinl-2011-200279

21. Fiorenza D, Viglio S, Lupi A, et al. Urinary desmosine excretion in acute exacerbations of COPD: A preliminary report. Respir Med. 2002;96(2):110-114.

doi: http://dx.doi.org/10.1053/rmed.2001.1224

22. Dahl R, Titlestad I, Lindqvist A, et al. Effects of an oral MMP-9 and-12 inhibitor, AZD1236, on biomarkers in moderate/severe COPD: A randomised controlled trial. Pulm Pharmacol Ther. 2012;25(2):169-177. doi: http://dx.doi.org/10.1016/j.pupt.2011.12.011

23. Stockley R, De Soyza A, Gunawardena K, et al. Phase II study of a neutrophil elastase inhibitor (AZD9668) in patients with bronchiectasis. Respir Med. 2013;107(4):524-533. doi: http://dx.doi.org/10.1016/j.rmed.2012.12.009

24. Lindberg CA, Engstrom G, de Verdier MG, et al. Total desmosines in plasma and urine correlate with lung function. Eur Respir J. 2012;39(4):839-845. doi: http://dx.doi.org/10.1183/09031936.00064611

25. Ongay S, Hendriks G, Hermans J, et al. Quantification of free and total desmosine and isodesmosine in human urine by liquid chromatography tandem mass spectrometry: $A$ comparison of the surrogate-analyte and the surrogate-matrix approach for quantitation. J Chromatogr A. 2014;1326:13-9. doi: http://dx.doi.org/10.1016/j.chroma.2013.12.035

26. Vestbo J, Anderson W, Coxson HO, et al. Evaluation of COPD longitudinally to identify predictive surrogate end-points (ECLIPSE). Eur Respir J. 2008;31(4):869-873. doi: http://dx.doi.org/10.1183/09031936.00111707 
27. Ma S, Lin YY, Turino GM. Measurements of desmosine and isodesmosine by mass spectrometry in COPD. Chest. 2007;131(5):1363-1371.

doi: http://dx.doi.org/10.1378/chest.06-2251

28. Slowik N, Ma S, He J, et al. The effect of secondhand smoke exposure on markers of elastin degradation. Chest. 2011;140(4):946-953.

doi: http://dx.doi.org/10.1378/chest.10-2298

29. Viglio S, Iadarola P, Lupi A, et al. MEKC of desmosine and isodesmosine in urine of chronic destructive lung disease patients. Eur Respir J. 2000;15(6):1039-1045. doi: http://dx.doi.org/10.1034/j.1399-3003.2000.01511.x

30. Ferrari F, Fumagalli M, Piccinini P, et al. Micellar electrokinetic chromatography with laser induced detection and liquid chromatography tandem mass-spectrometry-based desmosine assays in urine of patients with chronic obstructive pulmonary disease: A comparative analysis. J Chromatogr A. 2012;1266:103109. doi: http://dx.doi.org/10.1016/j.chroma.2012.10.014

31. Stone PJ, Gottlieb DJ, Oconnor GT, et al. Elastin and collagen degradation products in urine of smokers with and without chronic obstructive pulmonary-disease. Am J Respir Crit Care Med. 1995;151(4):952-959.

32. Vestbo J, Edward LD, Scanlon PD, et al. Changes in forced expiratory volume in 1 second over time in COPD. $N$ Eng $J$ Med. 2011; 365(13): 1184-1192.

doi: http://dx.doi.org/10.1056/NEJMoa1105482 